\title{
On the modeling of outflowing envelopes of massive evolved stars at arbitrary optical depths
}

\author{
Anton V. Dorodnitsyn and Gennadi S. Bisnovatyi-Kogan \\ Space Research Institute, \\ Profsoyuznaya Str. 84/32, Moscow 117997, Russia
}

Mass loss due to outflow is one factor introducing uncertainty into our understanding of the evolution of massive stars. There is a need of a theory, that would make it possible to take into account mass loss in the process of evolutionary computations in a self-consistent way. It is currently clear that the role of outflow is extremely important for stellar evolution, but quantitative conclusions about mass-loss rates remain uncertain. The evolution of stars with masses $M \geq 15 \mathrm{M}_{\odot}$ is accompanied by mass loss at rates reaching $10^{-4}-10^{-6} \mathrm{M}_{\odot} \mathrm{yr}^{-1}$. This strongly influences the evolution of such stars in the supergiant stage.

An equation of motion describing a spherically symmetrical, stationary outflow under the action of radiation pressure together with continuity equation reads:

$$
u \frac{d u}{d r}=-\frac{1}{\rho} \frac{d P_{g}}{d r}-\frac{\mathrm{GM}\left(1-\tilde{L}_{\mathrm{th}}\right)}{r^{2}}, \frac{\dot{M}}{4 \pi}=\rho u r^{2},
$$

where $\tilde{L}_{\text {th }}=L_{\text {th }}(r) / L_{e d}$ and $L_{\text {th }}=L_{\text {th }}(r) / L_{\text {ed }}$. The energy integral can be written in the form:

$$
L=4 \pi \rho u r^{2}\left(E+\frac{P}{\rho}-\frac{\mathrm{G} M}{r}+\frac{u^{2}}{2}\right)+L_{\mathrm{th}}(r) .
$$

Here $L$ is the constant total energy flux, which is made up of the fluxes of radiative energy and of the energy of the outflowing matter. The radiation flux $L_{\text {th }}$ can be found from the transport equation written in momentum form:

$$
L_{\mathrm{th}}=-\frac{4 \pi r^{2} c}{\kappa \rho}\left(\frac{d P_{r}}{d r}-\frac{E_{r} \rho-3 P_{r}}{r}\right) .
$$

$P_{r}$ is the radiation pressure and $E_{r}$ is the radiation energy density. The continuity equation, expressions for the pressure and energy density, and the optical depth can be written in the form:

$$
\begin{gathered}
P=P_{r}+P_{g}, \quad E=E_{r}+E_{g}+\epsilon_{i}, \\
P_{r}=\frac{a T^{4}}{3}\left(1-e^{-\tau}\right)+\frac{L_{\mathrm{th}}^{\infty}}{4 \pi r^{2} \mathrm{c}}, \quad E_{r} \rho=a T^{4}\left(1-e^{-\tau}\right)+\frac{L_{\mathrm{th}}^{\infty}}{4 \pi r^{2} \mathrm{c}}, \\
P_{g}=\rho \mathcal{R} T \quad, \quad E_{g}=\frac{3}{2} \mathcal{R} T .
\end{gathered}
$$




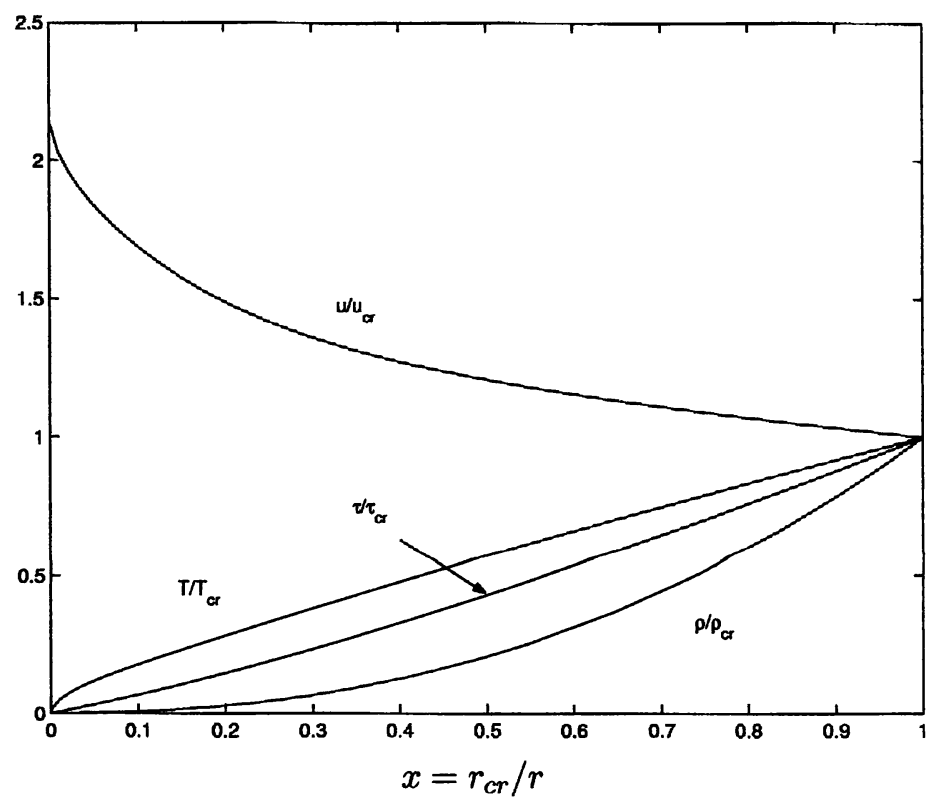

Figure 1. Distribution of velocity, density and optical depth. The sonic point is located at $x=1$, the second critical point is located at $x=0$. The curves are labeled with their respective ordinates.

Optical depth is determined by the following relation: $d \tau / d r=-\kappa \rho$. The obtained system of equations has two singular points: one is the sonic point and the other is situated at infinity $(T=0, \rho=0)$. The outer boundary condition is posed at infinity:

$$
T=0, \quad \rho \sim \frac{1}{r^{2}} \rightarrow 0, \quad u \rightarrow \text { const }=u_{\infty} .
$$

In the vicinity of the singular points analytical expansions are adopted. It can be shown that the solution at infinity follows asymptotic behaviour:

$$
\begin{gathered}
T=a_{\infty} \sqrt{x}, \quad \rho=b_{1} x^{2}+b_{2} x^{5 / 2}, \quad u \simeq u_{\infty}-\frac{b_{2}}{b_{1}^{2}} \sqrt{x}, \quad u_{\infty}=\frac{1}{b 1} . \\
\text { an expression for } v \text { reads: } \quad u \simeq u_{\infty}-\frac{b_{2}}{b_{1}^{2}} \sqrt{x}, \quad u_{\infty}=\frac{1}{b 1} .
\end{gathered}
$$

A numerical solution was found for the simplifying case of constant opacity and ionization degree. The two boundary value problem is solved using relaxation method. At vicinities of singular points a solution is represented via analytical expansions. Solution curves are depicted on the figure. This solution is for a $M=20 \mathrm{M}_{\odot}$ star, the following parameters at sonic point have been obtained: $T_{\mathrm{cr}}=6.04 \times 10^{2} \mathrm{~K}, r_{\mathrm{cr}}=7.88 \times 10^{15}, \rho_{\mathrm{cr}}=5.65 \times 10^{-15} \mathrm{~g} \mathrm{~cm}^{-3}, \tau_{\mathrm{ph}} \simeq 2$, $v_{\text {cr }} \simeq 2.75 \mathrm{~km} \mathrm{~s}^{-1}$, and $\dot{M} \simeq 1.5 \times 10^{-2} \mathrm{M}_{\odot} \mathrm{yr}^{-1}$.

\section{References}

Bisnovatyi-Kogan, G.S., Dorodnitsyn, A.V. 1999, A\&A 344, 647

Bisnovatyi-Kogan, G.S., Dorodnitsyn, A.V. 2001, Astron. Reports 45, 995 